\title{
Improvement In LEACH Protocol By Electing Master Cluster Heads To Enhance The Network Lifetime In WSN
}

\author{
Kanwalnainpreet Kaur \\ Lovely Professional University \\ Punjab, India
}

\author{
Deepika Sharma \\ Lovely Professional University \\ Punjab, India
}

\begin{abstract}
In wireless sensor networks, sensor nodes play the most prominent role. These sensor nodes are mainly un-chargeable, so it raises an issue regarding lifetime of the network. Mainly sensor nodes collect data and transmit it to the Base Station. So, most of the energy is consumed in the communication process between sensor nodes and the Base Station. In this paper, we present an improvement on LEACH protocol to enhance the network lifetime. Our goal is to reduce the transmissions between cluster heads and the sink node. We will choose optimum number of Master Cluster Heads from variation cluster heads present in the network. The simulation results show that our proposed algorithm enhances the network lifetime as compare to the LEACH protocol.
\end{abstract}

Keywords: Cluster, Cluster-head, Network Lifetime, sink node.

\section{INTRODUCTION}

In recent years, wireless sensor network (WSN) has achieved a great attention of the researchers. The network comprises of number of sensor nodes which are deployed according to required application. Sensor nodes mainly perform the three basic tasks namely, sensing, processing and transmitting. There are numerous applications of WSNs exists in number of directions which includes environmental applications, medical monitoring, security for homes, surveillance, inventory management industrial and manufacturing automation, process control, , distributed robotics, etc. Mainly the wireless sensor network is situated at any unreachable track. Once the sensor network has been established at particular location it may not be changed easily. The energy of sensor nodes is going to absorb when it collects and send the information. So the energy consumption by the sensor network has become the most attractive issue for the researchers [1].

All the sensor nodes are allowed to communicate through a wireless medium. Figure 1. describes the architecture of a senor node. The wireless medium may either of radio frequencies, infrared or any other medium, of course, having no wired connection.

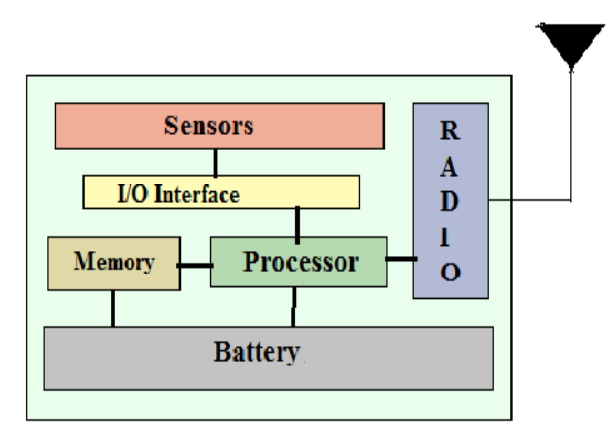

Figure 1. Architecture of sensor node

These nodes are deployed in a random fashion and they can communicate among themselves to make an ad-hoc network. WSN gives flexibility of adding nodes and removing the nodes as required. But this gives rise to many drastic changes to deal with in the network topology such as updating the path, or the network tree, etc. In a WSN the node that gathers the information refers to sink. The sink may be connected to the outside world through internet where the information can be utilized within time constraints [2].

The numbers of clustering algorithms have been proposed to improve the lifetime of the sensor network. In clustering, the sensor network is divided into clusters and then the one node from each cluster is selected as the cluster head. All the data aggregation activity has been done within the cluster and then cluster head use to send the information of a particular cluster to the BS which is also known as sink node. Clustering provides a reduction of redundancy and improvement over the lifetime of the wireless sensor network. LEACH is considered to be the most general clustering algorithm. In our proposed algorithm, an improvement over LEACH has been introduced. The simulation results show the improvement in the lifetime of the sensor network.

This paper is organized in the following manner. Section 2 is about literature survey regarding the variation clustering protocols of wireless sensor network. Section 3 will describes the block diagram representation of previous and new technique. Section 4 contains simulation results and the comparison of the new technique with the LEACH protocol. Finally, section 5 illustrates the conclusion for the work done for improving the lifetime of the network.

\section{LITERATURE SURVEY}

In this section, we present a brief study of some common clustering routing protocols for WSNs.

\subsection{Low Energy Adaptive Clustering Hierarchy Protocol for WSNs (LEACH)}

LEACH [3] is known as a distributed hierarchical protocol. It provides the aggregation for data in wireless sensor networks by selection of Cluster heads in random manner. This protocol first judges the strength of the received message or signal and then formation of cluster takes place. In this Cluster Head nodes are taken as routers to reach the sink node. Every nonCluster Head node sends its data to their CHs. Before sensing received information to sink, $\mathrm{CHs}$ aggregate the information. 
A stochastic algorithm is referred in each round by every single sensor node to determine whether it can be a cluster head for that particular round or may not act as a cluster head for that round. All normal nodes of the cluster communicate with $\mathrm{CH}$ in TDMA fashion which is scheduled by $\mathrm{CH}$. LEACH clustering is shown in Figure 2.

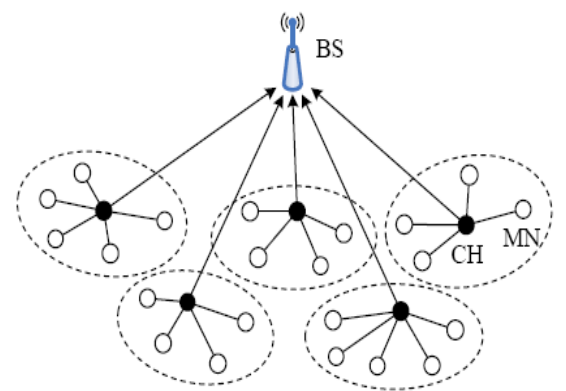

Figure 2. LEACH clustering

The operation of LEACH is conducted in numerous rounds, and each round is separated into two phases known as the setup phase and the steady-state phase. In the set-up phase the various clusters of sensor network are organized, while in the steady-state phase information is delivered to the sink node. During the set-up phase, each sensor node decides whether or not to act as a cluster head for that particular round. This decision is made by the sensor node by randomly selecting a number between 0 and 1 . A particular node becomes a cluster head for the current round if the number is less than the given following threshold value:

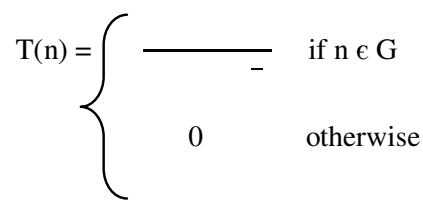

where $\mathrm{P}$ denotes desired percentage of cluster heads, $\mathrm{r}$ for current round, and $\mathrm{G}$ represents set of nodes that have not been elected $\mathrm{CHs}$ in the last 1/P rounds. When a sensor node is chosen as $\mathrm{CH}$ successfully, then it broadcasts an advertisement text to the other nodes of the network. By measuring the strength of received signal of the advertisement text, other sensor nodes decide to which cluster it will connect for this particular round and send a membership message to its $\mathrm{CH}$.

Another phase is steady-state phase. During this phase the nodes sense and transmit data to the cluster heads. The CHs aggregate the information/data arriving from sensor nodes that belong to the respective cluster, and send aggregated information to the sink node directly.

\subsection{Hybrid, Energy-Efficient, Distributed Clustering Approach (HEED)}

HEED [4] is also a distributed clustering algorithm used for Wireless Sensor Networks. Every sensor node has some amount of energy associated with it. This energy of nodes reduces during reception and transmission of data. It also access query requests coming from the Base Station. HEED protocol follows to circulate the role of server among all nodes of the cluster so that a balance will be maintained between residual energy of all nodes of the cluster. Hence, remaining energy of cluster head would not drop to minimum leading to less node failures due to energy depletion in the network.

\subsection{Power-Efficient Gathering in Sensor Information Systems (PEGASIS)}

In this protocol [5], some chains consisting of different sensor nodes have been formed. Every node sends its data to the neighbor sensor node and most appropriate node is selected to transmit the data to the sink node. PEGASIS does not follow the concept of cluster formation and it prefer to decide or choose only one node from the chain to transmit to the sink node instead of using multiple sensor nodes present in the network. When a sensor node fails due to low battery backup, again the chain is made using the same previous greedy approach.

\subsection{LEACH-C protocol}

LEACH-C [3] is a centralized clustering algorithm in which sink node has the power to select the clusters based upon the annealing algorithm to find $\mathrm{k}$ optimal number of clusters. Here the Base Station selects the cluster heads for a particular round. The protocol guarantees an optimum number of clusters but it has a drawback that each sensor node provides information about its current position and remaining energy to the sink node during the set up phase which results in an extra wastage or overhead in the network.

\subsection{LEACH-B}

LEACH-B [6] is an improvement in Leach protocol for wireless senor network. In order to minimize the energy consumption and to prolong the life span of the sensor network, the protocol needs to ensure that the partition of cluster should be balance and uniform. To achieve this goal, the number of $\mathrm{CHs}$ needs to be dominated, and the network needs an optimal $\mathrm{CHs}$ amount. At each round, after first selection of cluster head according to LEACH protocol, a second selection is introduced to modify the number of cluster head in consideration of node's residual energy.

\section{BLOCK DIAGRAM OF PREVIOUS AND PROPOSED TECHNIQUE}

\subsection{Previous technique- LEACH}

Figure 3. illustrates the basic block diagram of LEACH algorithm. It gives a representation of step by step operation of LEACH. The working initiates by deployment of senor nodes in a random manner in network. A traditional clustering scheme is applied to divide the sensor network into clusters. Then cluster heads are chosen randomly and transmit the data to Base station. This process continuous in every round until the whole sensor nodes die or Base station commands for stopping the process. 


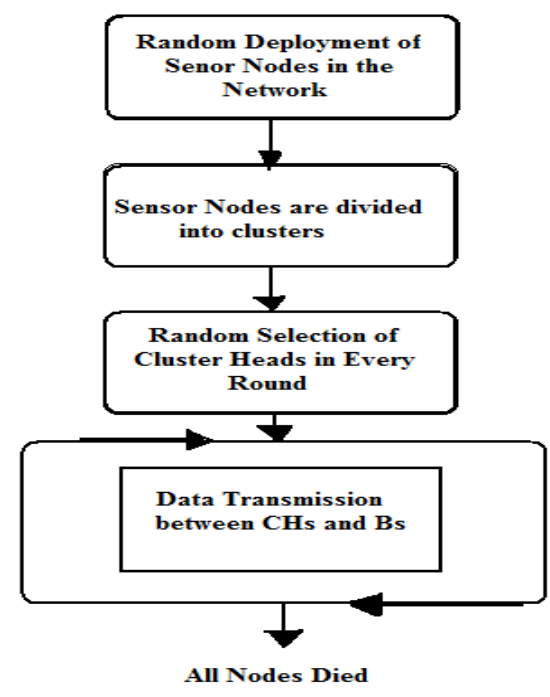

Figure 3. Basic Block Diagram for LEACH

\subsection{New Proposed Technique}

Figure 4. shows the basic block diagram for proposed technique. Every block marks the difference between the previous and new technique. Here, instead of transmission between every $\mathrm{CH}$ and sink node, it follows the technique of electing the Master Cluster Head for transmission of data from different cluster heads to the Base Station.

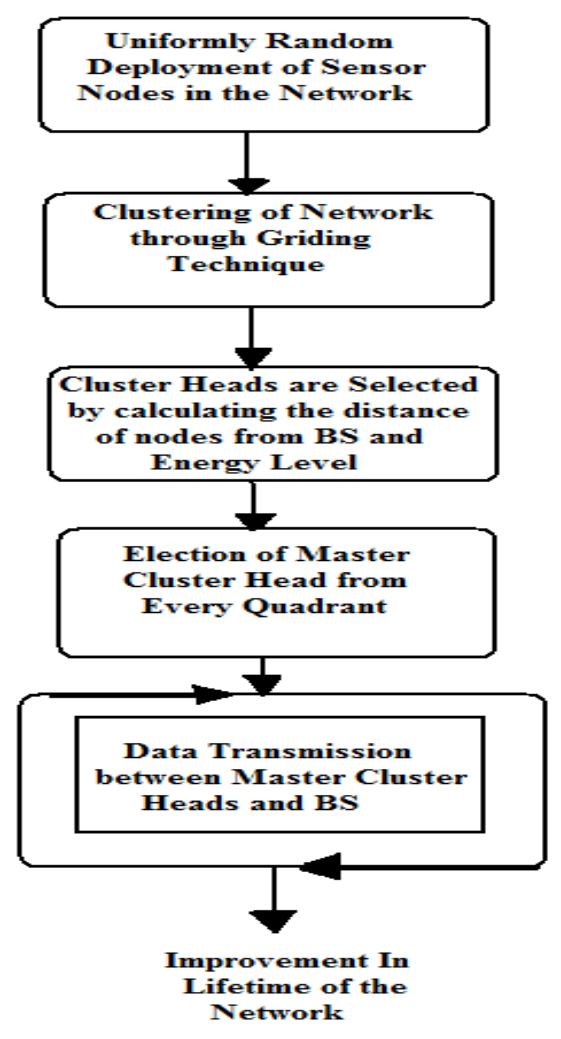

Figure 4.Basic Block Diagram for Proposed Technique
Thus our technique consists of following main stages:

- Random deployment of the nodes

- Formation of clusters using Gridding

- Selection of cluster heads and the intra-cluster communication has been made.

- Selection of Master Cluster Head from different cluster heads to minimize transmission process between $\mathrm{CHs}$ and sink node.

\section{Simulation And Analysis}

In this section, we perform simulations to analyze and evaluate the performance of the proposed technique. Here simulation is done on MATLAB. The simulation results depict that our proposed technique has better results in terms of the network lifetime. We get improvement in the lifetime of the network and with this more amount of information is gathered at the Base Station. To verify the improved algorithm proposed, we will compare the results with LEACH.

\subsection{Simulation Setup}

The different parameters which are used in our research has been mentioned in the Table I. We carried out our experiment using a simulation area of $100 \mathrm{~m} * 100 \mathrm{~m}$. The initial energy provided to every node is $0.5 \mathrm{~J}$. Every node use to send a data packet of 4000 bits.

TABLE I. Different values used during simulation

\begin{tabular}{|l|l|l|}
\hline Description & Symbol & Value \\
\hline The Sensing area & $M \times M$ & $100 \mathrm{~m} \times 100 \mathrm{~m}$ \\
\hline Number of nodes & $\mathrm{N}$ & 100 \\
\hline The initial node energy & $E_{\text {initial }}$ & $0.5 \mathrm{~J}$ \\
\hline $\begin{array}{l}\text { Energy consumed by } \\
\text { the amplifier to transmit } \\
\text { at a short distance }\end{array}$ & $E_{\mathrm{fs}}$ & $10 \mathrm{pJ} / \mathrm{bit} / \mathrm{m}^{2}$ \\
\hline $\begin{array}{l}\text { Energy consumed by } \\
\text { the amplifier to transmit } \\
\text { at a longer distance }\end{array}$ & $E_{a \mathrm{mp}}$ & $0.0013 \mathrm{pJ} / \mathrm{bit} / \mathrm{m}^{4}$ \\
\hline $\begin{array}{l}\text { Energy consumed in the } \\
\text { electronics circuit to } \\
\text { transmit or receive the } \\
\text { signal }\end{array}$ & $E_{\mathrm{elec}}$ & $50 \mathrm{pJ} / \mathrm{bit}$ \\
\hline $\begin{array}{l}\text { Data packet } \\
\text { Control packet }\end{array}$ & $\mathrm{k}$ & $4000 \mathrm{bits}$ \\
\hline $\begin{array}{l}\text { Data aggregationn } \\
\text { energy }\end{array}$ & $E_{\mathrm{da}}$ & $5 \mathrm{pJ} / \mathrm{bit} / \mathrm{report}$ \\
\hline
\end{tabular}

4.2 Deployment of sensor nodes and sink Here 100 sensor nodes are randomly deployed in the sensor field. The location of sink is $(50,140)$.

\subsection{Performance metrics}

There are number of parameters which affect the performance of the WSNs. The initial energy provided to each node is 
0.5J.This paper selects the two metrics that first node dies (FND), half of nodes die (HND) to evaluate the network performances. The network lifetime is depending on the number of alive nodes present. As the less number of nodes die, there will higher efficiency of using energy.

\subsection{Election of Master Cluster heads}

In this paper communication is take place between master cluster heads and sink node instead of cluster heads and sink node. Figure 5. shows simulation result of communication according to our technique.

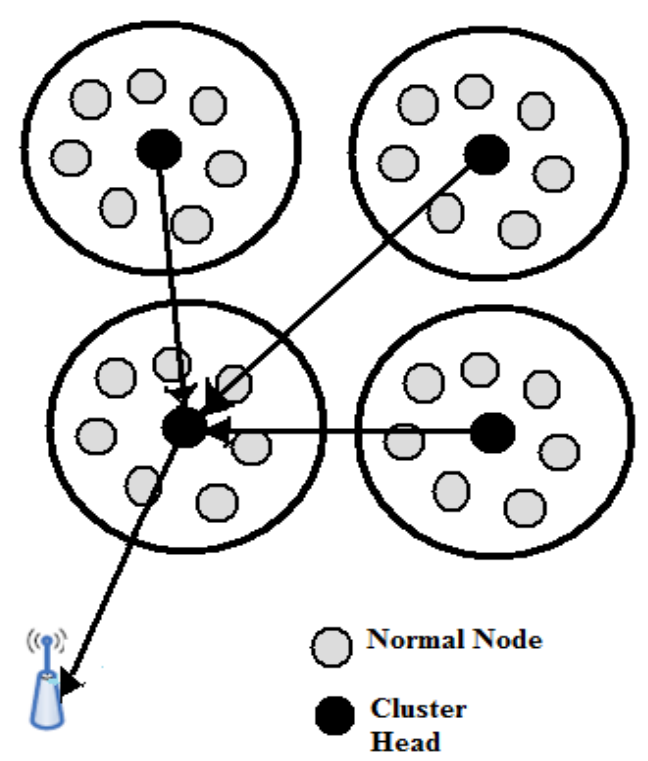

Figure 5. Master Cluster Head Technique

Every cluster head first selects its master cluster head and send its data to that particular node. These main nodes accept the data packets from cluster heads and then send aggregated data packets to the sink node. We can demonstrate from the figure that only one transmission is taking place. As we know that in sensor network most of the energy is consumed during the long way communication. So our main motive is to reduce the long way communication between cluster heads and the sink node. Our technique gives great improvement in the lifetime of the network.

\subsection{Result for lifetime of the network}

In Figure 6, the number of rounds is given on $x$-coordinate and $y$-coordinate represents for the number of dead nodes present per round. We calculated the FND and HND rounds for our technique. It can be demonstrate from the average results that first node dies around 1659 rounds and half nodes die at around 2226 rounds. Here we haven't taken external factors into consideration. The original LEACH algorithm began to its first node death at 1029th round and half node died in 1575 th round. So above results mark a great difference in our proposed technique as compare to LEACH.

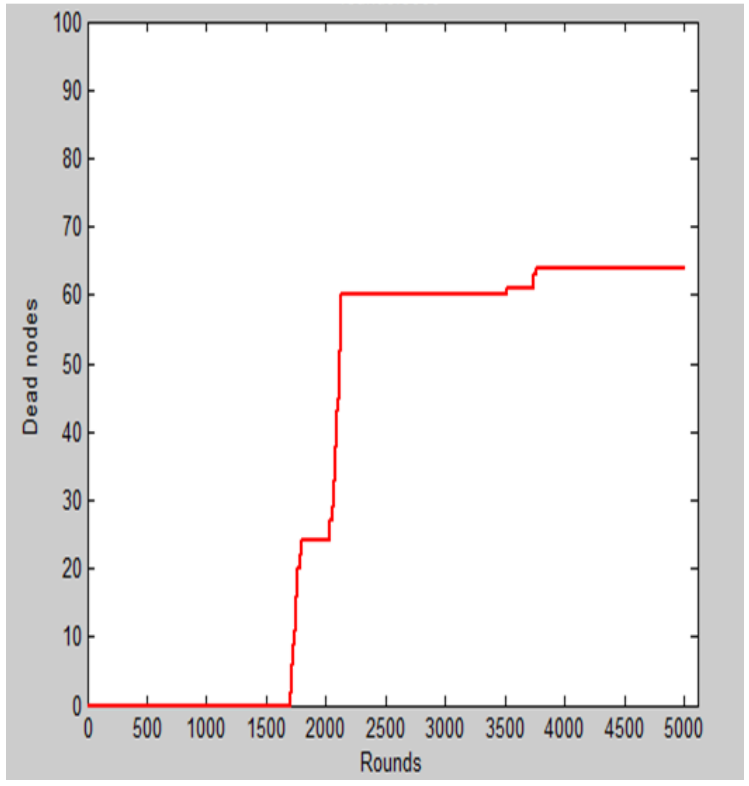

Figure 6. Network Lifetime of Proposed Technique

TABLE II. FND and HND for LEACH and Proposed Technique

\begin{tabular}{|l|l|l|}
\hline Techniques & FND & HND \\
\hline LEACH & 1029 & 1575 \\
\hline $\begin{array}{l}\text { Proposed } \\
\text { Technique }\end{array}$ & 1659 & 2226 \\
\hline
\end{tabular}

TABLE II shows the comparison of FND and HND between LEACH and new technique. If we define network lifetime as per FND and HND rounds, then new technique gives improved results as compare to LEACH.

\section{CONCLUSION}

The energy absorbed during transmission of information from senor nodes to sink node is becomes a most critical issue in WSN. We have to modify some present algorithms which will boosts the energy of the sensor nodes and so that the lifetime of the network will improved. In this paper, we modify a clustering algorithm known as LEACH and provide new clustering technique. It will improve the some disadvantages of LEACH. To minimize the load of the network, minimum number of cluster heads has been elected in each transmission round. The simulation results will show that our proposed protocol increases network lifetime

\section{ACKNOWLEDGMENTS}

First of all, I would like to thank Lovely Professional University for giving me a chance to work under this topic.

I would like to express my sincere gratitude to my project supervisor Ms. Deepika Sharma for her continuous support and encouragement. 
I would also like to express my deep gratitude to my father and mother for their care and support during my project. Without them the completion of this project would have been impossible.

\section{REFERENCES}

[1] Estrin D, Pisterk C D. "Connecting the physical word with pervasive networks". IEEE Pervasive Computing, 2002, pp.59-69.

[2] D.D.Chaudhary, S.P.Nayse, L.M.Waghmare (2011) "Application Of Wireless Sensor Networks For Greenhouse Parameter Control In Precision Agriculture", International Journal of Wireless \& Mobile Networks (IJWMN) Vol. 3, No. 1, 2011,pp. 140-149.

[3] Wendi Rabiner Heinzelman, Anantha Chandrakasan, and Hari Balakrishnan (2000), "Energy-Efficient Communication Protocol for Wireless Microsensor Networks", Proceedings of the 33rd Hawaii International Conference on System Sciences- 2000.

[4] Peyman Neamatollahi, Hoda Taheri, Mahmoud Naghibzadeh, Mohammad-Hossein Yaghmaee (2011) “A Hybrid Clustering Approach for Prolonging Lifetime in Wireless Sensor Networks" International symposium on computer networks and distributed systems,2011, pp.170-174.

[5] S. Lindsey and C.S. Raghavendra, .PEGASIS:Power efficient Gathering in Sensor Information System. Proceedings IEEE Aerospace,Conference, vol. 3, Big Sky, MT, Mar. 2002, pp. 1125-1130.

[6] Mu Tong and Minghao Tang, "LEACH-B: An Improved LEACH Protocol for Wireless Sensor Network" 2010 IEEE . 